\title{
On the Selection of the Redundant Carrier Positions in UW-OFDM
}

\author{
Heidi Steendam, Senior Member, IEEE
}

\begin{abstract}
Unique-word (UW) OFDM is a new multicarrier technique that was recently proposed in [M. Huemer, C. Hofbauer, and J. B. Huber, "The Potential of Unique Words in OFDM, Proc. InOWo, Hamburg, Germany, Sep. 2010, pp. 140-144]. In this technique, the guard interval that contains a priori known samples is part of the FFT interval. To be able to construct the UW-OFDM signal, some carriers must be sacrificed to transmit redundant information-these carriers are called the redundant carriers. It turns out that the average redundant energy needed strongly depends on the positions of the redundant carriers. In this paper, we look for the redundant carrier placement that minimizes the average redundant energy. However, this optimization is a NP-hard problem. Therefore, we reformulate the optimization problem and implement a branch and bound $(B \& B)$ algorithm to find the optimum. The results of the $B \& B$ search algorithm are compared to a theoretical lower bound, and with the results for two low cost redundant carrier placements, i.e., the quasi-uniform (QU) distribution [H. Steendam, "The Quasi-Uniform Redundant Carrier Placement for UW-OFDM," Vehicular Technology Conf., Fall 2012, Quebec, Canada, Sep. 2012], for the case where no guard bands are present, and the maximum distance (MD) distribution, in case forbidden guard bands are present. We show in this paper that when the full band is available for redundant carriers, both the redundant carrier placement found with the $B \& B$ algorithm and the $Q U$ distribution result in a redundant energy that is very close to the theoretical lower bound. Although the B\&B slightly outperforms the QU distribution, this implies that the QU distribution is a good low cost solution to the redundant carrier placement problem in the case of no guard bands. However, when there are forbidden guard bands in which the redundant carriers cannot be placed, the $Q U$ distribution can no longer be used. In that case, the results of the $B \& B$ algorithm are compared to that of the MD distribution. We show in this paper that the proposed $B \& B$ algorithm outperforms the MD distribution in the sense that it needs noticeably lower redundant energy.
\end{abstract}

Index Terms-Multicarrier, redundant energy, UW-OFDM.

\section{INTRODUCTION}

$\mathbf{T}$ HE multicarrier technique (as e.g., OFDM) is a technique that is suitable for high rate communication over dispersive channels [3]. This can be explained as the multicarrier technique splits the bandwidth in many narrow subchannels over which the data is transmitted in parallel. Because of the

Manuscript received April 06, 2012; revised August 08, 2012 and September 27, 2012; accepted November 27, 2012. Date of publication December 11, 2012; date of current version February 08,2013 . The associate editor coordinating the review of this manuscript and approving it for publication was Prof. Merouane Debbah. This work was supported by the Flemish Fund for Scientific Research (FWO).

The author is with the TELIN Department, Ghent University, Ghent B9000, Belgium (e-mail: Heidi.Steendam@telin.ugent.be)

Color versions of one or more of the figures in this paper are available online at http://ieeexplore.ieee.org.

Digital Object Identifier 10.1109/TSP.2012.2232657 narrow bandwidth of each subchannel, the channel behaves for each subchannel as a frequency-flat channel such that equalization becomes simple as compared to single carrier techniques. Because of this property, the multicarrier technique forms the basis of many wired and wireless standards, e.g., [4]-[7]. Intersymbol interference between successively transmitted multicarrier blocks is avoided by separating the different multicarrier blocks in the time domain by a guard interval. In the literature, several types of guard interval can be found [8]-[10], i.e., the cyclic prefix, zero padding, known symbol padding and time domain synchronous padding. Among these, the cyclic prefix is the most widespread. Common to these guard interval techniques is that the length of the multicarrier symbol is extended in the time domain. Recently, another technique was introduced, i.e., unique word (UW) OFDM [1], [11]. In this approach, in contrast with the other guard interval techniques, the guard interval is a part of the inverse fast Fourier transform (IFFT) block, i.e., the last samples of the IFFT block do not contain a data contribution but exist of known samples - the unique word. This specific structure of the UW-OFDM block, however requires that some of the carriers cannot transmit data, but have to transmit information that depends on the data symbols transmitted on the other carriers. Therefore, these carriers are called the redundant carriers.

Two methods to construct the UW-OFDM signal have been considered in the literature [12]: a direct approach, where the symbols transmitted on the redundant carriers not only depend on the data symbols that are transmitted on the data carriers, but also on the known samples from the unique word, and a two-step approach, where the redundant carrier symbols do not depend on the known samples. In this second technique, first the data part of the time-domain signal is generated with the IFFT, containing a block of zero samples at the positions of the unique word, and in the second step the known samples are added. This latter method is preferable, as it requires less redundant energy to be transmitted than the direct approach [12].

Although the UW-OFDM technique has a slightly lower throughput efficiency than CP-OFDM - this is because the guard interval is for UW-OFDM a part of the IFFT block whereas it is not for CP-OFDM - and the energy needed for the redundant carriers is non-negligible, it is shown in [12] for the uncoded case and in [1] and [13] for the coded case, that UW-OFDM outperforms CP-OFDM in terms of BER in the case of a dispersive channel. In an AWGN channel, however, the lower throughput efficiency of UW-OFDM will cause a small degradation as compared to CP-OFDM. The explanation for the success of UW-OFDM can be found by taking a closer look at the structure. The UW-OFDM time-domain signal contains a block of zeros in the time domain. This requires 
the introduction of redundancy in the frequency domain, i.e., the redundant carriers. In that sense, UW-OFDM is equivalent to a Reed-Solomon code [14]. The receiver for UW-OFDM acts like a decoder - despite the required redundant energy, a receiver fully exploiting the redundancy is able to transform it into a coding gain. The coding gain turns out to be larger when the dispersive channel contains more deep fades. This implies that in channels with deep fades, UW-OFDM is more favorable than CP-OFDM. Note that precoding is able to mitigate the effect of deep fades in CP-OFDM [15]-[17]. However, to obtain optimal performance, this requires channel state information (CSI) to be available at the transmitter side, which is not obvious to obtain in a sufficiently accurate and timely manner. In contrast, UW-OFDM does not need CSI.

The performance gain in UW-OFDM comes at a price: the receiver will be more complex than the simple linear data detector for CP-OFDM. Further, one of the main differences between CP-OFDM and UW-OFDM is the content of the guard interval. In CP-OFDM, the guard interval samples depend on the data symbols and are therefore not a priori known at the receiver, whereas in UW-OFDM, the receiver knows the content of the guard interval. Therefore, the guard interval in UW-OFDM can be used more easily for parameter estimation. In that sense, UW-OFDM is comparable to known symbol padding (KSP) OFDM, where it has been shown in [18] that because of the known samples from the guard interval, channel estimation and synchronization in KSP-OFDM is more accurate than in CP-OFDM. Further, UW-OFDM has lower out-of-band emission than traditional OFDM systems [11].

In [19], the effect of the redundant carrier placement on the redundant energy is studied for equidistantly spaced redundant carriers and the split distribution. It is shown that the energy needed for the redundant carriers strongly depends on the positions of the redundant carriers - when these positions are not appropriately chosen, the redundant energy explodes, as e.g., with the equidistant distribution. To keep the redundant energy low, the redundant carrier positions have to be selected carefully. The split distribution, which was further studied in [20], has a redundant energy that is still far from the theoretical lower bound. The search for the optimal placement of the redundant carriers that minimizes the average redundant energy started in [2]. Unfortunately, this is a NP-hard combinatorial optimization problem, such that an exhaustive search becomes prohibitively computationally complex for even small values for the FFT size and the number of redundant carriers. Based on the exhaustive search for small values of the FFT size, the quasi-uniform (QU) distribution was introduced in [2]. Although the QU distribution is suboptimal, its corresponding redundant energy is already close to the theoretical lower bound, such that it is a good low cost solution to select the positions of the redundant carriers. However, when the OFDM spectrum contains guard bands in which the redundant carriers cannot be placed, the QU distribution cannot be used. To solve the redundant carrier placement problem in this case, we go back to the combinatorial optimization problem. In this paper, we reformulate the optimization problem to obtain a related optimization problem that can be implemented with acceptable computational cost using a branch and bound (B\&B) algorithm, and determine its performance for the cases with and

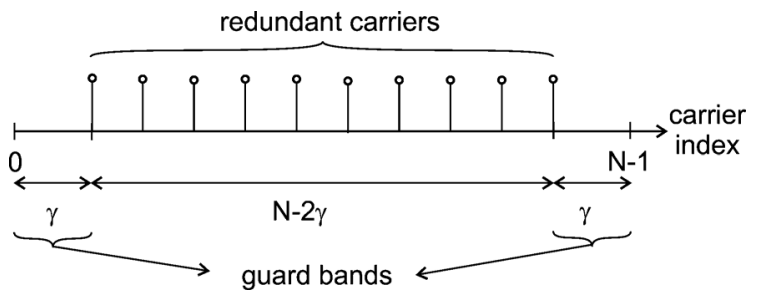

Fig. 1. Position of the guard bands.

without guard bands. We show in the paper that in more than $97 \%$ of the cases, this algorithm is able to find with a low computation cost the optimum, and for the cases where the optimum was not found, the resulting redundant energy was very close to the minimum. For the forbidden guard band case, we consider the maximum distance (MD) distribution, a low cost distribution similar to the QU distribution, that is based on a pilot carrier placement studied in [21]. This distribution maximizes the distance between the different redundant carriers, taking into account the presence of forbidden carrier positions, as e.g., shown in Fig. 1. Note that when no guard bands are present, the maximum distance distribution reduces to the quasi-uniform distribution. We show in this paper that the B\&B algorithm outperforms the MD distribution, although for large FFT size, the MD distribution turns out to be an acceptable suboptimal solution.

\section{THE UW-OFDM SYSTEM}

In UW-OFDM, the transmitted signal consists of blocks of $N$ samples that are generated at a rate $1 / T$. Each block of samples can be decomposed into two parts: the first $N-N_{u}$ samples contain the data part, and the $N_{u}$ last samples are the unique word with prior known samples. The time-domain structure is shown in Fig. 2. To obtain the UW-OFDM time-domain signal, first the data part is generated with an inverse FFT, such that the last $N_{u}$ samples are zero. This requires that $N_{r} \geq N_{u}$ redundant carriers have to be modulated with a linear combination of the data symbols transmitted on the data carriers. The time-domain samples of the data part can be written as

$$
\mathbf{y}=\mathbf{F}_{N}^{-1} \mathbf{P}\left(\begin{array}{c}
\mathbf{x}_{d} \\
\mathbf{x}_{r}
\end{array}\right)
$$

where the $N_{d} \times 1$ vector $\mathbf{x}_{d}=\left[x_{d}(1) \ldots x_{d}\left(N_{d}\right)\right]^{T}$ contains the $N_{d}=N-N_{r}$ data symbols and the $N_{r} \times 1$ vector $\mathbf{x}_{r}=$ $\left[x_{r}(1) \ldots x_{r}\left(N_{r}\right)\right]^{T}$ the redundant symbols. The $N \times N$ permutation matrix $\mathbf{P}$ defines the positions of the data and redundant carriers, and the $N \times N$ IFFT matrix is given by $\left(\mathbf{F}_{N}\right)_{k, \ell}=$ $\frac{1}{\sqrt{N}} e^{-j 2 \pi \frac{k \ell}{N}}$. We decompose the permutation matrix in two submatrices $\mathbf{P}=\left[\begin{array}{ll}\mathbf{P}_{d} & \mathbf{P}_{r}\end{array}\right]$, where $\mathbf{P}_{d}\left(N \times N_{d}\right)$ and $\mathbf{P}_{r}\left(N \times N_{r}\right)$ determine the data and redundant carrier positions, respectively. The sets $I_{d}$ and $I_{r}$ contain the data carrier positions and redundant carrier positions, such that the columns of $\mathbf{P}_{d}$ and $\mathbf{P}_{r}$ consist of unit-weight vectors with a ' 1 ' at the positions $\tilde{n}_{\ell} \in I_{d}$ and $n_{\ell} \in I_{r}$, respectively. In the second step, the unique word is added to $\mathbf{y}$.

The $N_{r}$ redundant symbols $\mathbf{x}_{r}$ consist of a linear combination of the $N-N_{r}$ data symbols $\mathbf{x}_{d}$ :

$$
\mathbf{x}_{r}=-\mathbf{M}_{22}^{\dagger} \mathbf{M}_{21} \mathbf{x}_{d}=\mathbf{T} \mathbf{x}_{d},
$$




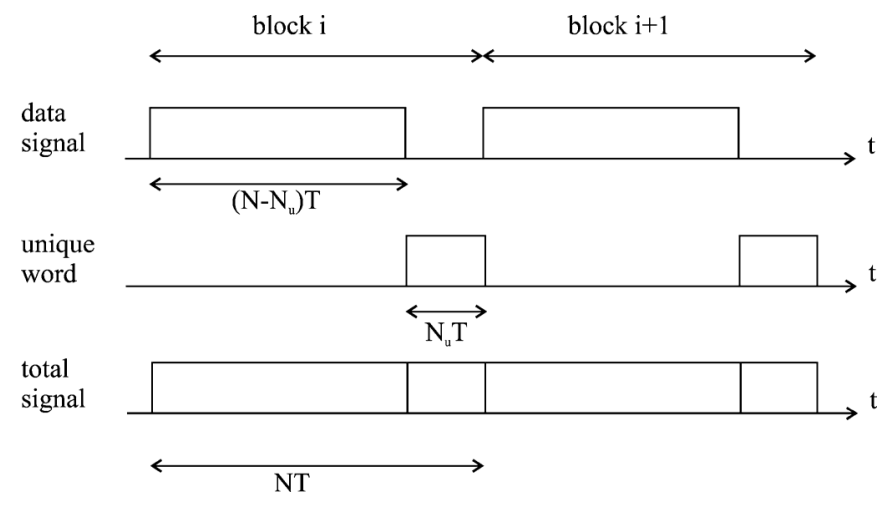

Fig. 2. Time-domain signal of UW-OFDM.

where $\mathbf{M}_{22}^{\dagger}=\mathbf{M}_{22}^{H}\left(\mathbf{M}_{22} \mathbf{M}_{22}^{H}\right)^{-1}$ is the Penrose-Moore pseudo-inverse, and $\mathbf{M}=\mathbf{F}_{N}^{-1} \mathbf{P}$ is decomposed as

$$
\mathbf{M}=\left(\begin{array}{ll}
\mathbf{M}_{11} & \mathbf{M}_{12} \\
\mathbf{M}_{21} & \mathbf{M}_{22}
\end{array}\right)
$$

with $\mathbf{M}_{11}$ is $\left(N-N_{u}\right) \times N_{d}, \mathbf{M}_{12}$ is $\left(N-N_{u}\right) \times N_{r}, \mathbf{M}_{21}$ is $N_{u} \times N_{d}$ and $\mathbf{M}_{22}$ is $N_{u} \times N_{r}$. Hence, the time domain signal (1) can be rewritten as

$$
\mathbf{y}=\mathbf{M}\left(\begin{array}{c}
\mathbf{I}_{N_{d}} \\
\mathbf{T}
\end{array}\right) \mathbf{x}_{d}=\mathbf{G} \mathbf{x}_{d},
$$

with $\mathbf{I}_{N_{d}}$ is the $N_{d} \times N_{d}$ identity matrix. Note that the last $N_{u}$ rows in $\mathbf{G}$ are zero rows.

The average energy of the time-domain signal $\mathbf{y}$ is given by $P_{t}=E\left[\mathbf{y}^{H} \mathbf{y}\right]=P_{d}+P_{r}$. The average data energy equals $P_{d}=N_{d} E_{s}$, where $E\left[x_{d}(i) x_{d}^{*}(j)\right]=E_{s} \delta_{i, j}$ is the energy of a data symbol. The average redundant energy can be rewritten as $P_{r}=E\left[\mathbf{x}_{r}^{H} \mathbf{x}_{r}\right]=E_{s}$ trace $\left(\mathbf{T}^{H} \mathbf{T}\right)$. Taking into account the definition of $\mathbf{T}(2)$, this results in

$$
P_{r}=E_{s} \operatorname{trace}\left[\mathbf{M}_{21} \mathbf{M}_{21}^{H}\left(\mathbf{M}_{22} \mathbf{M}_{22}^{H}\right)^{-1}\right],
$$

where we have used the property trace $(\mathbf{A B C})=\operatorname{trace}(\mathbf{C A B})$ [22]. The matrices $\mathbf{M}_{21} \mathbf{M}_{21}^{H}$ and $\mathbf{M}_{22} \mathbf{M}_{22}^{H}$ from (5) are Hermitian Toeplitz matrices with as elements

$$
\begin{aligned}
& \left(\mathbf{M}_{21} \mathbf{M}_{21}^{H}\right)_{k, k^{\prime}}=\frac{1}{N} \sum_{\ell=0}^{N_{d}-1} e^{j 2 \pi \frac{\left(k-k^{\prime}\right) \tilde{n}_{\ell}}{N}} \\
& \left(\mathbf{M}_{22} \mathbf{M}_{22}^{H}\right)_{k, k^{\prime}}=\frac{1}{N} \sum_{\ell=0}^{N_{r}-1} e^{j 2 \pi \frac{\left(k-k^{\prime}\right) n_{\ell}}{N}}
\end{aligned}
$$

where $k, k^{\prime}=0, \ldots, N_{u}-1$. Taking into account that $I_{r} \cup$ $I_{d}=\{0, \ldots, N-1\}$, it can easily be verified that $\mathbf{M}_{21} \mathbf{M}_{21}^{H}+$ $\mathbf{M}_{22} \mathbf{M}_{22}^{H}=\mathbf{I}_{N_{u}}$.

In [2], it is shown that a cyclic shift of all carrier positions has no influence on the average redundant energy. Further, a theoretical lower bound on the average redundant energy was derived in [20], resulting in the average redundant energy

$$
P_{r, L B}=E_{s} \frac{N_{u} N_{d}}{N_{r}} .
$$

This lower bound requires the matrices $\mathbf{M}_{21} \mathbf{M}_{21}^{H}$ and $\mathbf{M}_{22} \mathbf{M}_{22}^{H}$ to be diagonal matrices. As it is not certain that this requirement can be achieved by any redundant carrier placement, it is a theoretical lower bound on the average redundant energy. In [20], it is analytically shown that when $N_{r}$ is a power of 2, a uniform distribution of the redundant carriers over the bandwidth is optimal, and the lower bound on the average redundant energy is reached. The indices of the redundant carriers are in this case given by $n_{\ell}=n_{0}+\ell \Delta, \ell=1 \ldots N_{r}-1$, where $0 \leq n_{0}<\Delta$ and $\Delta=N / N_{r}$. However, when $N_{r}$ is not a power of 2 , it was shown in [19] that this equidistant distribution can result in a high average redundant energy, as in some cases the matrix $\mathbf{M}_{22} \mathbf{M}_{22}^{H}$ in (5) will become (close to) singular.

\section{OPTIMAL Redundant CARRIER Placement}

Finding the redundant carrier placement that minimizes the redundant energy is an NP-hard integer combinatorial optimization problem. Hence, an exhaustive search over all possible redundant carrier positions is prohibitively complex, even for small values of $N$ and $N_{r}$. Therefore, we consider a reformulation of the optimization problem to obtain an algorithm with a computation time that is polynomial in $N$ and $N_{r}$. The goal of this section is to make use of algebraic manipulation and linear programming techniques to generate a limited set of redundant carrier placements out of which the optimal placement can be found, instead of considering all possible redundant carrier placements in the optimization.

Let us first rewrite the average redundant energy (5). Defining $\mathbf{A}=\mathbf{M}_{22} \mathbf{M}_{22}^{H}$ and taking into account that $\mathbf{M}_{21} \mathbf{M}_{21}^{H}+\mathbf{M}_{22} \mathbf{M}_{22}^{H}=\mathbf{I}_{N_{u}}$, the average redundant energy is equal to

$$
P_{r}=E_{s} \operatorname{trace}\left(\mathbf{A}^{-1}\right)-E_{s} N_{u} .
$$

Hence, minimizing the average redundant energy is equivalent with minimizing trace $\left(\mathbf{A}^{-1}\right)$. Note that $\mathbf{A}$ is a Hermitian Toeplitz matrix with as elements $\mathbf{A}_{k, k^{\prime}}=a_{k-k^{\prime}}$, where

$$
a_{k}=\frac{1}{N} \sum_{\ell=1}^{N_{r}} e^{j 2 \pi \frac{k n_{\ell}}{N}} .
$$

Let us assume that the matrix $\mathbf{A}$ has eigenvalues $\lambda_{m}, m=$ $1, \ldots, N_{u}$. Because of the Hermitian nature of $\mathbf{A}$, the eigenvalues are real valued. Further, $\mathbf{A}$ and $\mathbf{I}_{N_{u}}-\mathbf{A}$ are positive semi definite matrices, such that $0 \leq \lambda_{m} \leq 1$. Taking this into account, trace $\left(\mathbf{A}^{-1}\right)=\sum_{m=1}^{N_{u}} \frac{1}{\lambda_{m}}$. Hence, to minimize the redundant energy, the following optimization problem should be solved:

$$
\begin{aligned}
& \min _{\left\{n_{\ell}\right\}} \sum_{m=1}^{N_{u}} \frac{1}{\lambda_{m}} \\
& \text { subject to } n_{\ell} \in\{0, \ldots, N-1\} .
\end{aligned}
$$

In theory, when the eigenvalues could take all values (no conditioning on $n_{\ell}$ ), the average redundant energy is minimized when all eigenvalues are equal: $\lambda_{m}=\frac{N_{r}}{N}$. This implies that the matrix $\mathbf{A}$ is a diagonal matrix, and results in the theoretical lower bound from the previous section. 
Let us look closer at the optimization problem (11). First note that the function $f(x)=\frac{1}{x}$ is a strictly convex function for $x>0$, i.e., the second derivative $f^{\prime \prime}(x)>0$. Hence, the function to be minimized in (11) is a strictly convex function. This implies that the minimum corresponding to $\lambda_{m}=\frac{N_{r}}{N}$ is a unique minimum. Hence, for general $N, N_{r}$ and $N_{u}$, the eigenvalues corresponding to the minimum redundant energy will be in the neighbourhood of this unique minimum, i.e., the eigenvalues will all be close to $\lambda_{m} \approx \frac{N_{r}}{N}$, resulting in a small eigenvalue spread $\lambda_{\max }-\lambda_{\min }$, where $\lambda_{\max }$ and $\lambda_{\min }$ are the largest and smallest eigenvalue of $\mathbf{A}$, respectively. Therefore, instead of minimizing (11), we look for the redundant carrier placements that have a small eigenvalue spread

$$
\begin{aligned}
& \min _{\left\{n_{\ell}\right\}}\left(\lambda_{\max }-\lambda_{\min }\right) \\
& \text { subject to } n_{\ell} \in\{0, \ldots, N-1\} .
\end{aligned}
$$

Gerschgorin [22] defined bounds on the eigenvalues of a matrix by deriving a set of circles in the complex plane, in which the eigenvalues must be located. Applying the Gerschgorin theory to the matrix $\mathbf{A}$, we find the following set of circles:

$$
\left|z-a_{0}\right| \leq r_{i}
$$

where

$$
r_{i}=\sum_{\substack{j=1 \\ j \neq i}}^{N_{u}}\left|a_{i-j}\right|
$$

Taking a closer look at these Gerschgorin circles, it turns out that they define a set of concentric circles around $z=a_{0}=$ $\frac{N_{r}}{N}$. Further, taking into account that the eigenvalues of $\mathbf{A}$ are real-valued, it follows that the set of Gerschgorin circles reduce to a set of intervals on the real axis: $\frac{N_{r}}{N}-r_{i} \leq \lambda_{i} \leq \frac{N_{r}}{N}+$ $r_{i}$. Hence, the minimum and maximum eigenvalue of $\mathbf{A}$ are bounded by:

$$
\begin{aligned}
\lambda_{\min } & \geq \frac{N_{r}}{N}-r_{\max } \\
\lambda_{\max } & \leq \frac{N_{r}}{N}+r_{\max }
\end{aligned}
$$

where $r_{\max }=\max r_{i}$. If we can make this maximum radius $r_{\text {max }}$ as small as possible, the eigenvalue spread will also be small. So, we reformulate the optimization problem (12) to make $r_{\max }$ as small as possible, which results in a minimax problem on $r_{i}$ :

$$
\begin{aligned}
& \min _{\left\{n_{\ell}\right\}} \max _{i} r_{i}, \quad i=1, \ldots, N_{u} \\
& \text { subject to } n_{\ell} \in\{0, \ldots, N-1\} .
\end{aligned}
$$

Assume that the eigenvector corresponding to eigenvalue $\lambda_{m}$ is given by $\mathbf{x}_{m}$. Because of the Hermitian nature of $\mathbf{A}$, its eigenvector matrix $\mathbf{X}=\left(\mathbf{x}_{1} \ldots \mathbf{x}_{N_{u}}\right)$ is an orthogonal matrix. This implies that $\sum_{m=1}^{N_{u}} x_{m}(k) x_{m}^{*}\left(k^{\prime}\right)=\delta_{k, k^{\prime}}$ and $\sum_{k=1}^{N_{u}} x_{m}(k) x_{m^{\prime}}^{*}(k)=\delta_{m, m^{\prime}}$. Further, as $\mathbf{A}=\mathbf{X} \mathbf{\Lambda} \mathbf{X}^{H}$, where $\boldsymbol{\Lambda}=\operatorname{diag}\left\{\lambda_{m}\right\}$, the components $a_{k-k^{\prime}}$ of the matrix $\mathbf{A}$ can be rewritten as

$$
a_{k-k^{\prime}}=\sum_{m=1}^{N_{u}} \lambda_{m} x_{m}(k) x_{m}^{*}\left(k^{\prime}\right) .
$$

Let us consider the following sum:

$$
\sum_{k^{\prime}=1}^{N_{u}}\left|a_{k-k^{\prime}}\right|^{2}=\sum_{m=1}^{N_{u}} \lambda_{M}^{2}\left|x_{m}(k)\right|^{2} \leq \lambda_{\max }^{2} .
$$

Hence, the sum of squared moduli of a row of $\mathbf{A}$ is upper bounded by $\lambda_{\max }^{2}$, i.e., the largest eigenvalue. Rewriting (18) results in

$$
\sum_{\substack{k^{\prime}=1 \\ k^{\prime} \neq k}}^{N_{u}}\left|a_{k-k^{\prime}}\right|^{2} \leq \lambda_{\max }^{2}-a_{0}^{2}
$$

In the case of the minimum redundant energy, the maximum eigenvalue $\lambda_{\max }$ will be close to $a_{0}=\frac{N_{r}}{N}$. Hence the right hand side in (19) will be small, such that the non-diagonal values of $\mathbf{A}$ must have a small amplitude for the optimal redundant carrier placement. This implies that for redundant carrier placements that have a redundant energy that is close to the minimum, the different $r_{i}$ will be close to each other. Therefore, instead of considering the minimax problem on $r_{i}$, we restrict our attention to the minimization of $r_{1}=\sum_{k=1}^{N_{u}-1}\left|a_{k}\right|$ :

$$
\begin{aligned}
& \min _{\left\{n_{\ell}\right\}} \sum_{k=1}^{N_{u}-1}\left|a_{k}\right| \\
& \text { subject to } n_{\ell} \in\{0, \ldots, N-1\} .
\end{aligned}
$$

Let us take a closer look at the minimization of $r_{1}$. This problem can be rewritten as

$$
\begin{aligned}
\min _{\left\{n_{\ell}\right\}} \sum_{k=1}^{N_{u}-1}\left|a_{k}\right| & =\min _{\left\{n_{\ell}\right\}} \sum_{k=0}^{N_{u}-1}\left|a_{k}\right| \\
& =\min _{\left\{n_{\ell}\right\}} \sum_{k=0}^{N_{u}-1}\left|a_{k}\right|^{2} \\
& =\min _{\left\{n_{\ell}\right\}} \sum_{k=0}^{N_{u}-1}\left|\frac{1}{N} \sum_{\ell=1}^{N_{r}} e^{j 2 \pi \frac{k n_{\ell}}{N}}\right|^{2} \\
& =\min _{\left\{n_{\ell}\right\}} \sum_{\ell, \ell^{\prime}=1}^{N_{r}} g\left(n_{\ell}-n_{\ell^{\prime}}\right)
\end{aligned}
$$

where

$$
g(x)=\cos \frac{\pi\left(N_{u}-1\right) x}{N} \cdot \frac{\sin \frac{\pi N_{u} x}{N}}{\sin \frac{\pi x}{N}} .
$$

In (21a), we have added the constant $a_{0}=\frac{N_{r}}{N}$, and (21b) follows from the fact that $x^{2}$ is a monotonically increasing function for $x>0$. In (21c), we have inserted the definition of $a_{k}(10)$, and (21d) follows straightforwardly from the rearrangement of (21c). Note that $g(x)$ is a symmetric function: $g(-x)=g(x)$ and $g(N-x)=g(x)$. As the redundant carrier position $n_{\ell}$ is an integer between 0 and $N-1, n_{\ell}-n_{\ell^{\prime}}$ is an integer in the interval $-(N-1) \leq n_{\ell}-n_{\ell^{\prime}} \leq N-1$. Hence, (21d) is a function of the relative differences of the redundant carrier positions only. The function (22) is shown in Fig. 3 for different values of $N_{u}$. 


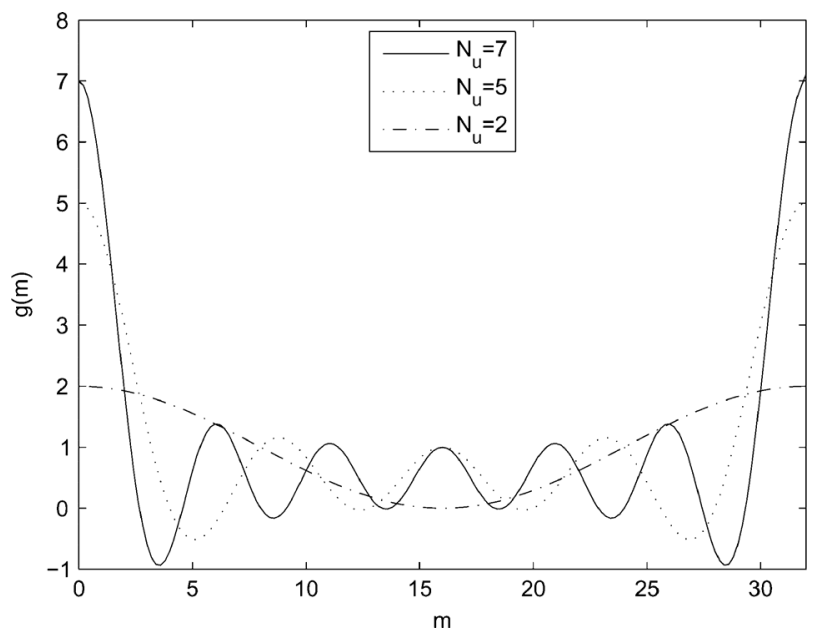

Fig. 3. The function $g(m), N=32$.

Taking into account the symmetry of $g(x)$, the sum in (21d) can be rewritten as

$$
\sum_{\ell, \ell^{\prime}=1}^{N_{r}} g\left(n_{\ell}-n_{\ell^{\prime}}\right)=2 \sum_{\ell=1}^{N_{r}} \sum_{\ell^{\prime}=1}^{\ell-1} g\left(n_{\ell}-n_{\ell^{\prime}}\right)+N_{r} g(0) .
$$

Defining

$$
S_{\ell}=\sum_{\ell^{\prime}=1}^{\ell-1} g\left(n_{\ell}-n_{\ell^{\prime}}\right)
$$

and neglecting the irrelevant constants, the minimization of (21d) results in

$$
\min _{\left\{n_{\ell}\right\}} \sum_{\ell, \ell^{\prime}=1}^{N_{r}} g\left(n_{\ell}-n_{\ell^{\prime}}\right)=\min _{\left\{n_{\ell}\right\}} \sum_{\ell=1}^{N_{r}} S_{\ell} .
$$

Hence, we finally reformulate the optimization problem into

$$
\begin{aligned}
& \min _{\left\{n_{\ell}\right\}} \sum_{\ell=1}^{N_{r}} S_{\ell} \\
& \text { subject to } n_{\ell} \in\{0, \ldots, N-1\} .
\end{aligned}
$$

Although the optimization problems (11), (12), (16), (20) and (26) are not strictly equivalent in a mathematical sense, it follows from the derivations that redundant carrier placements that have a small $S=\sum_{\ell=1}^{N_{r}} S_{\ell}$, will also have a small eigenvalue spread for the matrix $\mathbf{A}$, and thus also a redundant energy that is close to the minimum. Therefore, we can use (26) to generate a limited set of redundant carrier placements, out of which the optimal placement that minimizes (11) can be found.

Taking into account that $S$ is a linear combination of the function $g(\cdot)$, it is clear that optimization of (25) can be achieved with a branch and bound algorithm, which is a technique to tackle integer linear programming problems [23]. The first step in this technique is branching. In this step, we gradually build up a search tree by adding in each level of the tree an additional redundant carrier position. In a full tree, all possible redundant carrier placements correspond to a path in the tree from the root to an end node. For a node at level $i$ in the tree, corresponding to the set of positions $\left\{n_{1}, \ldots, n_{i}\right\}$, the path metric equals $\sum_{\ell=1}^{i} S_{\ell}$. Our goal is to find the paths (redundant carrier placements) with the lowest path metric $S$ at the end nodes. The number of end nodes increases exponentially with $N$ and $N_{r}$, so the tree must be pruned to reduce the computational complexity of the search algorithm. This step is called bounding. The two keywords are to prune the tree as early as possible, i.e., eliminating a branch at level 1 reduces the number of end nodes more than eliminating a branch at level $N_{r}-1$, and to prune the tree in an intelligent way. If we do not prune in an intelligent way, we risk to lose the optimal solution.

The search algorithm that we have used to construct and prune the tree in an intelligent way is given in Table I, and takes into account the presence of forbidden guard bands $\left(I_{\mathrm{GB}}\right.$ is the set of forbidden carrier positions, if no guard bands are present $\left.I_{\mathrm{GB}}=\varnothing\right)$. The algorithm contains three parameters: $K, M$ and $\alpha$. With the parameter $K$, we reduce the number of candidates for the newly added position $n_{i}$ at level $i-1$ of the tree: only the positions with the lowest branch metric $f(y)$ (see Table I for the definition of $f(y)$ ) are considered for further processing. This limits the growth of the tree at each level. The parameter $M$ determines the number of paths in the tree that is kept for the next level and avoids the number of paths in the tree to grow too fast. If $M$ is selected too small, the risk exists that paths are eliminated at an intermediate level that have at the end level a lower metric than the paths that are not eliminated. Hence, if $M$ is too small, we might miss the optimal solution. However, increasing $M$ will result in a higher computational load. On the other hand, the optimal choice for $K$ is correlated to the shape of $g(m)$. If $g(m)$ slowly varies as function of $m$ (as for smaller values for $N_{u}$ ), larger values for $K$ are needed than when $g(m)$ has a sharp peak (as for $N_{u} \approx N_{r}$, see also Fig. 3). When $g(m)$ slowly varies, adding the next carrier position becomes more difficult: the path metrics will be close to each other, i.e., the minimum is very broad. To find the optimum in that case, not only $K$ must be larger, but typically also $M$ must increase. To improve the convergence speed in that case, we introduced the third parameter $\alpha$. By replacing $g(m)$ by

$$
\tilde{g}(m)=\operatorname{sign}(g(m)) \cdot|g(m)|^{\alpha}
$$

with $\alpha>0$, we artificially adapt the shape of the function $g(m)$, to increase the difference between the path metrics and obtain a better discrimination between the different carrier positions that can be added at a level, so that a smaller value for $M$ for the algorithm to converge is required. As $\operatorname{sign}(x) \cdot|x|^{\alpha}$ is a convex function for $\alpha>0$, it has no influence on the solution of the minimization problem:

$$
\min _{\left\{n_{\ell}\right\}} \sum_{\ell=1}^{N_{r}} \sum_{\ell^{\prime}=1}^{\ell-1} \tilde{g}\left(n_{\ell}-n_{\ell^{\prime}}\right) \equiv \min _{\left\{n_{\ell}\right\}} \sum_{\ell=1}^{N_{r}} \sum_{\ell^{\prime}=1}^{\ell-1} g\left(n_{\ell}-n_{\ell^{\prime}}\right) .
$$

Another issue that is related to the convergence speed is the presence of duplicate redundant carrier placements in the tree. Before we add a new redundant carrier position to a path, we have to check if the resulting set $\left\{n_{1}, \ldots, n_{i}\right\}$ is not already present in the tree, in the same or a different order. If this is the case, we would add a path that has the same properties as the other path in the tree. As such copies of sets will require a higher $M$ to converge to the same result, we should avoid to add 
TABLE I

The SEARCh Algorithm

1) compute $\tilde{g}(m), m=1, \ldots, N$

2) initialize tree $=\left[n_{1} \notin I_{G B}, \Sigma_{0}=0\right]$

3) for $i=2: N_{r} \%$ level $i$ is added

4) treenew=empty; $x=\#$ rows in tree

5) for $k=1: x$

6) $\quad\left\{n_{1}, \ldots, n_{i-1}\right\}=\operatorname{tree}(k ; 1: i-1)$

7) define $f(y)=\sum_{\ell=1}^{i-1} \tilde{g}\left(y-n_{\ell}\right)$

8) select the $K$ values with smallest $f\left(n_{i}^{j}\right)$

with $n_{i}^{j} \notin\left\{n_{1}, \ldots, n_{i-1}\right\} \cup I_{G B}, j=1, \ldots, K$

for $j=1: K$

test if $\left\{n_{1}, \ldots, n_{i-1}, n_{i}^{j}\right\} \subset$ tree

if not: add carrier position

$\Sigma_{i}=\Sigma_{i-1}+f\left(n_{i}^{j}\right)$

treenew $=\left[\right.$ treenew $\left.;\left\{n_{1}, \ldots, n_{i-1}, n_{i}^{j}\right\}, \Sigma_{i}\right]$

end

end

end

sort treenew on $\Sigma_{i}$ and select $M$ best ones to put in tree end

TABLE II

COMPLEXITY OF THE ALGORITHM

\begin{tabular}{l|c|c}
\hline \multicolumn{1}{c|}{ task } & action & $\#$ actions \\
\hline 1. compute $g(x)$ & LU & $3 N$ \\
& MUL & $2 N$ \\
\hline \multirow{2}{*}{ 2. run B\&B } & ADD & $\mathcal{O}\left(\frac{\left(N+K^{2}\right)}{2} N_{r}^{2}\right)$ \\
& SORT & $\left(N_{r}-1\right)\left[N \log _{2} N+K M \log _{2} K M\right]$ \\
\hline 3. compute $P_{r}$ & LU & $M\left(N_{u}-1\right) N_{r}$ \\
& EIG & $M \mathcal{O}\left(N_{u}^{3}\right)$ \\
& DIV & $M N_{u}$ \\
& ADD & $M\left(N_{u}-1\right)+2 M\left(N_{r}-1\right)$ \\
& SORT & $M \log _{2} M$ \\
\hline
\end{tabular}

with $\mathrm{LU}=$ lookup operation, $\mathrm{MUL}=$ real valued multiplication, $\mathrm{ADD}=$ real valued addition, DIV $=$ real valued division, SORT $=$ sorting, $\mathrm{EIG}=$ eigenvalue computation

such duplicate paths to the tree. This is tested in line 10 of the algorithm.

The algorithm delivers $M$ sets of redundant carrier positions, out of which the set must be found that minimizes the average redundant energy. The complexity of the algorithm is discussed in Table II, where for the main subtasks of the algorithm (task $1=$ initialization by computing $f(x)$, task $2=$ building up the search tree for the B\&B algorithm, and task $3=$ selecting out of the candidate sequences the one with the smallest redundant energy) the number of multiplications, additions, etc. is given. To compute the eigenvalues of the $N_{u} \times N_{u}$ matrices in the last part of the algorithm we consider the complexity $\mathcal{O}\left(N_{u}^{3}\right)$, although in reality, it is possible to obtain the eigenvalues with lower complexity, as the matrices are Hermitian Toeplitz matrices. However, the exact computational complexity of such algorithms cannot be expressed with a simple expression in $N_{u}$. The exact complexity of the last part of the algorithm turns out to be not that important, as the slowest part of the algorithm is the $\mathrm{B} \& \mathrm{~B}$ part: the complexity of this part increases non-linearly with the parameter $M$, which is typically several orders of magnitude larger than the other parameters. As a comparison, for an exhaustive search over all possible sequences, we have to consider the third part of the proposed algorithm only (the computation of $\left.P_{r}\right)$, but with $M$ replaced by $\frac{N !}{N_{r} !\left(N-N_{r}\right) !}$. It is obvious that the complexity of the proposed algorithm is much lower than that of the exhaustive search. On the other hand, the redundant carrier positions according to the QU distribution and the MD distribution can be computed using simple analytical expressions, as e.g., for the QU distribution: $n_{\ell}=\operatorname{round}\left(\ell N / N_{r}\right)$. Hence, compared to the $\mathrm{QU}$ and $\mathrm{MD}$ distribution, the $\mathrm{B} \& \mathrm{~B}$ algorithm has higher computational complexity.

\section{NumERICAL RESULtS}

In the simulations, we considered the guard band locations given in Fig. 1. The two guard bands of width $\gamma$ carriers are located at the two edges of the frequency band, so in total $2 \gamma$ carriers cannot be used for placing redundant carriers. Note that the case $\gamma=0$ corresponds to the case without guard bands.

First, we want to evaluate the redundant carrier placements that are delivered by the branch and bound algorithm. For 156 cases with $N \in\{16,32\}$ and different values for $N_{r}$ and $N_{u}$, we have computed the optimal redundant carrier placement through an exhaustive search. For the same cases, we used the branch and bound algorithm to find the optimal redundant carrier placement, where the parameters were selected out of the sets $M \in\{100,1000\}, K \in\{3,5,10,15,20,25\}$ and $\alpha \in\{0.5,1,2, \ldots, 10\}$. In 152 of the cases, the branch and bound algorithm found the optimal placement of the redundant carriers, corresponding to a success rate of more than $97 \%$. In the cases where the optimum placement was not found, the B\&B algorithm returned a set of redundant carrier positions with a redundant energy that was maximum $5 \%$ above the optimum. Most of the convergence problems occurred when $N_{r}$ was a power of 2. However, in that case, the optimum placement is known: it is the uniform distribution. By combining the results from the $\mathrm{B} \& \mathrm{~B}$ algorithm with the known optima, only in one case, the optimum was not found, resulting for that case in a redundant energy that was only $0.00021 \%$ above the optimal redundant energy. Hence, the proposed B\&B algorithm combined with the known optima is able to find the optimal redundant carrier placement in virtually all considered cases.

Next, the results from the $\mathrm{B} \& \mathrm{~B}$ algorithm (with $\gamma=0$ ) are compared to the results for the quasi-uniform distribution from [2] for 221 cases with $N \in\{16,32\}$ and the same values for $M, K$ and $\alpha$ from the previous test. Only in four cases, the quasi-uniform distribution resulted in a lower average redundant energy than the redundant carrier placement delivered by the $\mathrm{B} \& \mathrm{~B}$ algorithm. In these cases, the redundant energy for the quasi-uniform distribution was equal to the theoretical lower bound, implying that the quasi-uniform distribution was the optimal distribution. The B\&B algorithm in those cases resulted in a redundant energy that was only slightly higher than this optimum. In all other cases, the redundant energy for the B\&B algorithm was lower than or equal to that of the quasi-uniform distribution. A similar test was carried out for the case where guard bands are present $(\gamma>0)$. When $2 \gamma>\left\lceil\frac{N}{N_{r}}\right\rceil$, i.e., for the cases where the MD distribution does not reduce to the QU distribution, the MD distribution always resulted in a redundant energy that was (much) larger than that corresponding to the carrier placement delivered by the B\&B algorithm.

In only a few cases, the best solution was not found with the $B \& B$ algorithm with the parameter sets used in the two tests. By increasing $M$ and $K$, in the limit the optimum will be found 
nonetheless as the pruned tree will converge to the full tree, which of course contains the optimum. However, this comes at the price of an increased computational complexity. For most of the tests, $\alpha=1$ yielded a good result, only when $N_{u} \ll N_{r}$, other values of $\alpha$ were necessary to obtain the optimum or a result that was better than that of the quasi-uniform distribution. For $N_{u} \approx N_{r}, M=100$ and $K \in\{3,5,10\}$ already gave the best result. When $N_{u} \ll N_{r}$, the required $M$ and $K$ increased to $M=1000$ and $K \in\{15,20,25\}$. This effect was also described in the previous section: when $N_{u} \approx N_{r}$, the function $g(m)$ has a sharp peak, such that there is a good discrimination between the different carrier positions out of which the next carrier position must be chosen. Only a few positions will compete with each other, resulting in a fast convergence. When $N_{u} \ll N_{r}$, the minimum is very broad, so the choice for the next carrier position to be added becomes more difficult, with as consequence a slower convergence, even when $\alpha \neq 1$ was used.

In the two tests, for each simulation, we first had to look for the values of $K, M$ and $\alpha$ that resulted in the lowest redundant energy. The selection of the optimal parameters in the algorithm is not straightforward, as three parameters have to optimized. The strategy that is used was the following. First, we selected $M \gtrsim 10 N$ and $\alpha=1$, and determined the value of $K$ that results in the lowest redundant energy. Next, for that value of $K$ and $\alpha=1$, we increased $M$ until no further reduction in the redundant energy occurred. For the obtained $K$ and $M$, we finally looked for the value of $\alpha$ that minimized the redundant energy. In this way, the three-dimensional parameter optimization is split into three one-dimensional searches, first over $K$, then over $M$ and finally over $\alpha$. However, based on the simulations carried out in the two tests, we could determine some simple rules of thumb for the selection of the parameters, that resulted in a close to optimal redundant energy. First of all, $\alpha=1$ yielded in the majority of the cases $(>90 \%)$ the best result. Hence, the optimization over $\alpha$ could be omitted. Secondly, selecting $M$ of the order of $10-100 \times N$ always resulted in a redundant energy that was very close to the theoretical lower bound. Thirdly, in most cases, a small value of $K$, e.g., $K=5$ or $K=10$, yielded a redundant energy that was close to the theoretical lower bound. Hence, as a conclusion, the parameters of the algorithm resulting in a close to optimal performance are $\alpha=1, K=5$ or $K=10$, and $M$ is of the order of $10-100 \times N$.

In the following, we define $P_{r, X}$ with $X=$ $\{Q U, B \& B, M D, L B\}$ as the average redundant energy corresponding with the quasi-uniform distribution, the redundant carrier placement delivered by the $\mathrm{B} \& \mathrm{~B}$ algorithm, the maximum distance distribution, and the theoretical lower bound, respectively. In Fig. 4, the relative gain in redundant energy when comparing the carrier placement of the $\mathrm{B} \& \mathrm{~B}$ algorithm with the quasi-uniform distribution is shown. As can be observed, the quasi-uniform distribution exhibits a redundant energy that is up to $3 \%$ larger than the redundant carrier placement from the $\mathrm{B} \& \mathrm{~B}$ algorithm. In Fig. 5, the average redundant energy for the quasi-uniform distribution and the result from the B\&B algorithm are shown relatively to the theoretical lower bound. The results from the $\mathrm{B} \& \mathrm{~B}$ algorithm in this figure corresponded to the optimum

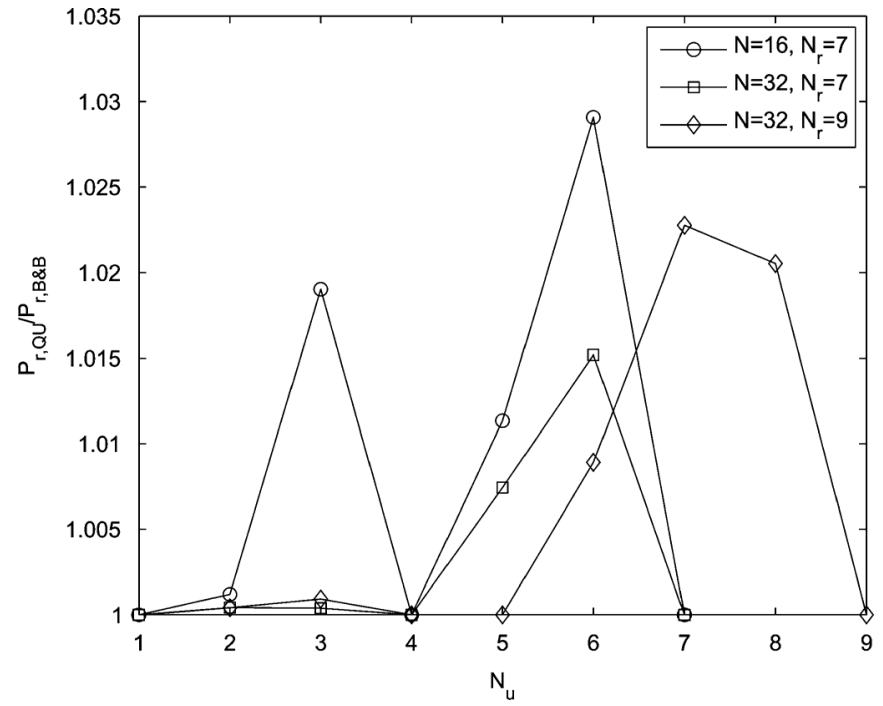

Fig. 4. Relative redundant energy gain of the B\&B carrier placement as compared to the quasi-uniform distribution $(\gamma=0)$.

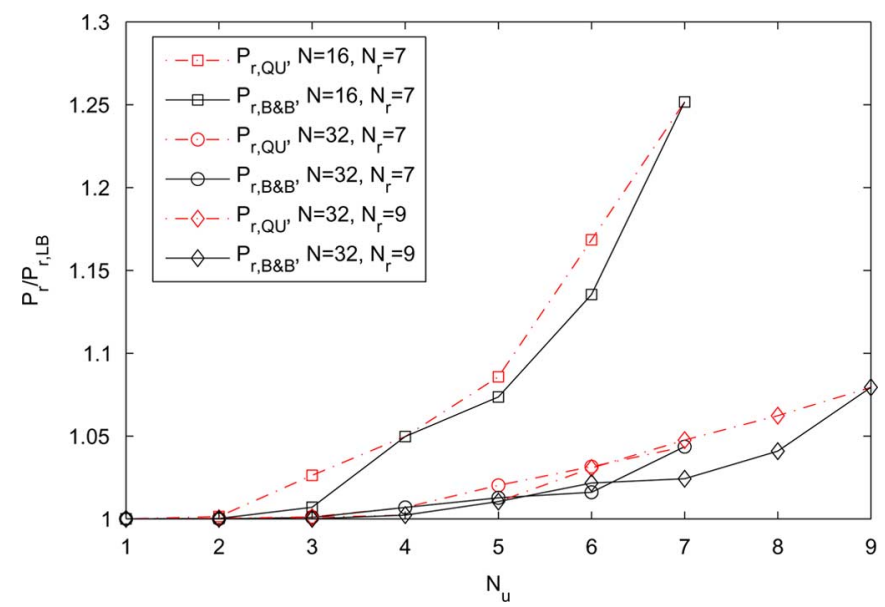

Fig. 5. Redundant energy compared to the theoretical lower bound, no guard bands $(\gamma=0)$.

distribution obtained with the exhaustive search. It can clearly be observed that the theoretical lower bound cannot be reached in practice, especially for small values of $N$ and $N_{u} \approx N_{r}$. The worst case corresponds to $N_{r}=N_{u}$ : in that case, the difference between the redundant energy and its theoretical lower bound is the largest, not only for the cases in the figure, but for all cases considered in the simulations. In Fig. 6, we assume the presence of guard bands. The average energy for the maximum distance distribution is compared to that of the B\&B algorithm. Similarly as in Fig. 5, the average energy is shown relative to the theoretical lower bound. The first thing that we can observe is that the average redundant energy in the case of forbidden guard bands is increased as compared to the case of no guard bands. Further, the curves for the $\mathrm{B} \& \mathrm{~B}$ algorithm are always lower than the curves for the MD distribution. Also, the relative difference between the two curves is larger than for the no guard bands case in Fig. 5. Hence, while for the case of no guard bands, the low cost QU solution is a good alternative for the B\&B algorithm, as it has quasi optimal performance, the low cost MD distribution for 


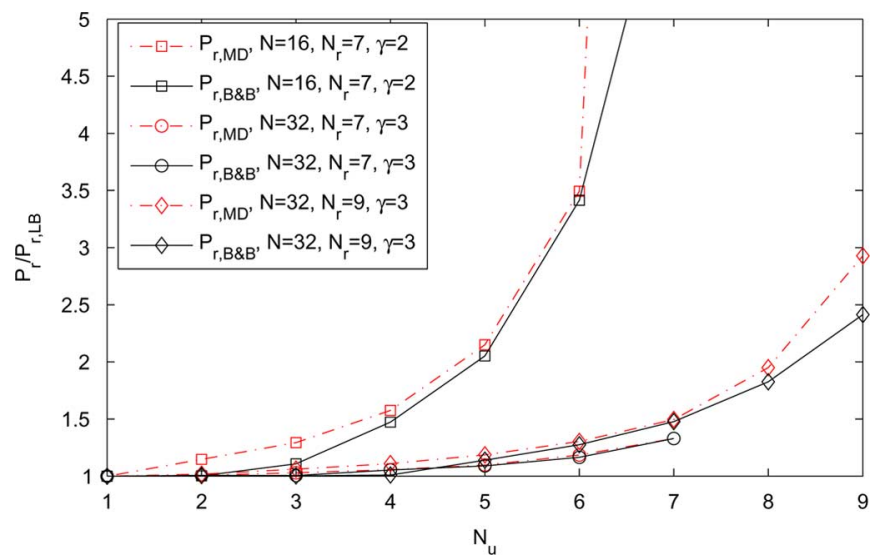

Fig. 6. Redundant energy compared to the theoretical lower bound, with guard bands, $\gamma=8$.

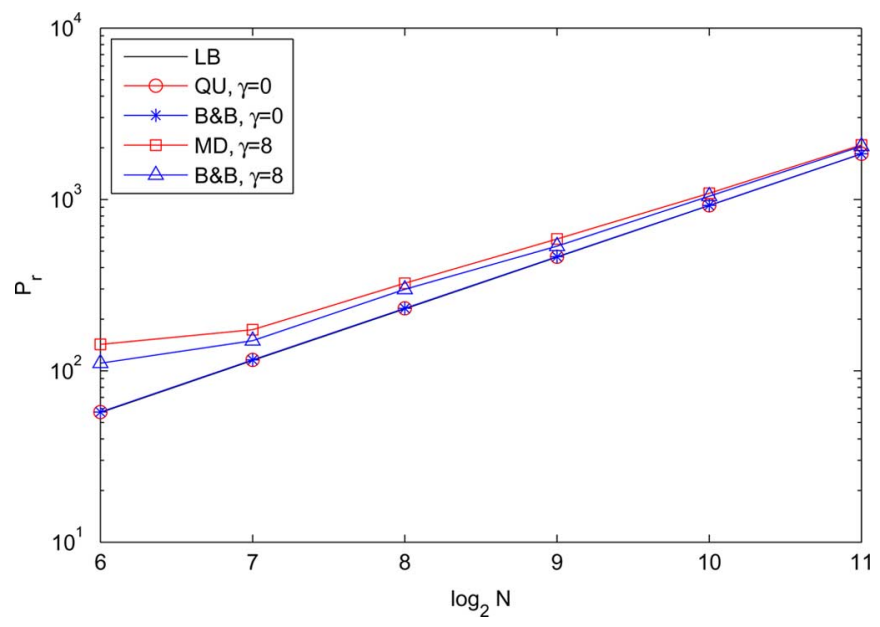

Fig. 7. Redundant energy for $N_{u}=N_{r}, N_{r}=\lceil 0.1 N\rceil, E_{s}=1$.

the forbidden guard band case performs not that well. In that case, we should use the proposed B\&B algorithm.

From the previous tests, it followed that the proposed B\&B algorithm is able to deliver a redundant carrier placement that is if not optimal, then at least close to optimal for small $N$. We now consider the case of larger $N$. In Fig. 7, the redundant energy is shown for larger values of $N$ when $N_{r}=N_{u}$ and the number of redundant carriers equals about $10 \%$ of the FFT size. The curves for the quasi-uniform distribution and the B\&B algorithm with $\gamma=0$ perfectly coincide and are close to the theoretical lower bound, whereas for $\gamma=8$, the resulting redundant energy is higher. The MD distribution does not perform as well as the B\&B algorithm. Also for all other simulations that we carried out, we found that for $N_{r}=N_{u}$ and $\gamma=0$, the best carrier placement from the B\&B algorithm was the quasi-uniform distribution. This strongly indicates that the quasi-uniform distribution is optimum for $N_{r}=N_{u}$. This can be intuitively explained as follows. From the tests of the algorithm, it turned out that for $N_{u} \approx N_{r}$, sorting the possible sets on the absolute value of their path metric, i.e., on $\left|\Sigma_{i}\right|$, and selecting the $K$ values with smallest $\left|f\left(n_{i}^{j}\right)\right|$ gave better convergence, whereas for small $N_{u}$, the convergence of the algorithm is better when we just sort on $\Sigma_{i}$ and $f\left(n_{i}^{j}\right)$. Hence, this corresponds to placing

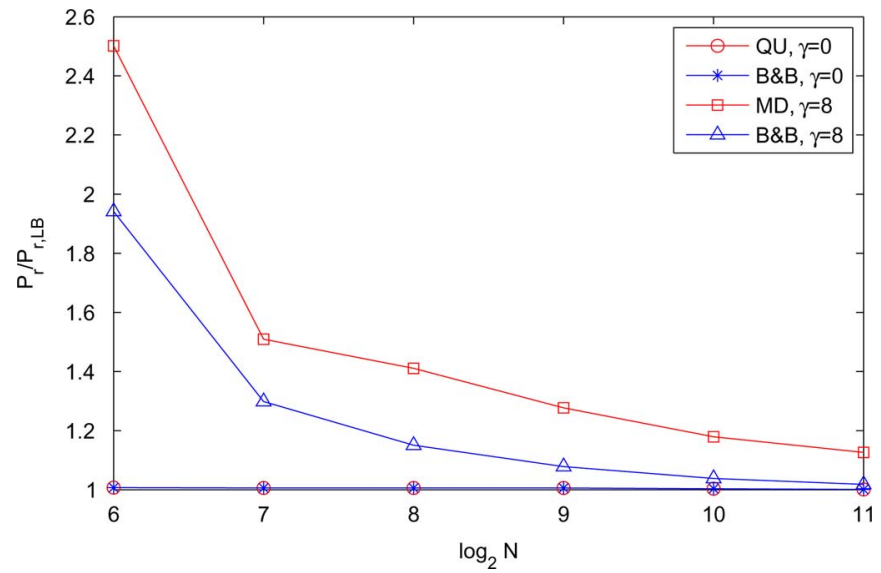

Fig. 8. Relative difference of the redundant energy with the lower theoretical bound for $N_{u}=N_{r}, N_{r}=\lceil 0.1 N\rceil$.

the redundant carriers close to the zero crossings in the function $g(x)$ (22). For $N_{r}=N_{u}$, these zero crossings are given by $x=$ $\frac{N}{N_{r}-1}(0.5+k-1)$ and $x=\frac{N}{N_{r}} k, k=1, \ldots, N_{r}-1$. Note that by selecting the redundant carrier positions $n_{\ell}=$ round $\left(\frac{N}{N r} \ell\right)$ as in the quasi-uniform distribution, the relative differences between the different carrier positions correspond to an integer value that is closest to one of these zero crossings.

In Fig. 8, we show the relative difference of the redundant energy from the QU, MD and B\&B carrier placements with the theoretical lower bound. The curves for the QU and B\&B carrier placement $(\gamma=0)$ are very close to the theoretical lower bound. The difference between the curves for the MD distribution and the $\mathrm{B} \& \mathrm{~B}$ algorithm with $\gamma=8$ is larger, although the difference decreases with increasing $N$. Also, the gap between the cases with guard band and the theoretical lower bound decreases when the FFT size increases. This indicates that for large $N$, the low cost MD solution is a good suboptimal alternative for the redundant carrier placement, at the cost of a small increase in redundant energy as compared to the carrier placement from the B\&B algorithm. However, when $N \leq 1024$, the B\&B algorithm turns out to be a better choice to determine the redundant carrier positions.

\section{CONCLUSION}

In this paper, we have reformulated the NP-hard combinatorial optimization problem to find the redundant carrier placement that minimizes the average redundant energy in UW-OFDM. As a consequence, we were able to use a branch and bound algorithm to reduce the complexity of the optimization. In this paper, we have shown that the proposed algorithm is able to find the optimal carrier placement in the majority of the cases with reasonably low computational cost, and for the cases where the optimum was not found, the resulting carrier placement had a redundant energy that was close to the optimum. In the special case $N_{r}=N_{u}$, and in the absence of guard bands, the tests with the $\mathrm{B} \& \mathrm{~B}$ algorithm indicate that the quasi-uniform distribution is the optimum carrier placement. For $N_{u}<N_{r}$, the branch and bound algorithm yielded in most cases a redundant carrier placement that had lower redundant 
energy than the quasi-uniform distribution, although the difference is small as the quasi-uniform distribution already gave a result that was close to the theoretical lower bound. Hence, when the full band is available for placing redundant carriers, the low cost quasi-uniform distribution is a good low cost solution for the redundant carrier placement problem.

In the case that the bandwidth of the UW-OFDM system contains guard bands where no information may be transmitted on the carriers in that guard band, we might not be able to put the redundant carriers according to the quasi-uniform distribution. In that case, we proposed the low cost maximum distance distribution. However, compared to the results of the B\&B algorithm, the MD distribution requires a redundant energy that is noticeable higher. For large values of $N(N>1024)$, the difference between the two techniques reduces, such that in that case, the low cost MD distribution is an acceptable technique to select the redundant carrier positions, at the cost of a limited redundant energy increase. For smaller values of $N$, the $\mathrm{B} \& \mathrm{~B}$ algorithm clearly outperforms the MD distribution.

\section{ACKNOWLEDGMENT}

The author wants to thank Prof. Veerle Fack for the discussion on branch and bound algorithms.

\section{REFERENCES}

[1] M. Huemer, C. Hofbauer, and J. B. Huber, "The potential of unique words in OFDM," in Proc. InOWo, Hamburg, Germany, Sep. 2010, pp. $140-144$.

[2] H. Steendam, "The quasi-uniform redundant carrier placement for UW-OFDM," presented at the Veh. Technol. Conf. (VTC) Fall 2012, Quebec, Canada, Sep. 2012.

[3] J. A. C. Bingham, "Multicarrier modulation for data transmission, an idea whose time has come," IEEE Commun. Mag., vol. 31, pp. 5-14, May 1990.

[4] Transmission and Multiplexing (TM); Access Transmission Systems on Metallic Access Cables; Very High Speed Digital Subscriber Line (VDSL); Part 2: Transceiver Specification, ETSI TS 101 270-2, 2001.

[5] Digital Video Broadcasting (DVB); Framing Structure, Channel Coding and Modulation For Digital Terrestrial Television, ETSI EN 300744 V1.6.1 (2009-01), 01/2009.

[6] R. van Nee, G. Awater, M. Morikura, H. Takanashi, M. Webster, and K. W. Halford, "New high-rate wireless LAN standards," IEEE Commun. Mag., vol. 37, pp. 82-88, Dec. 1999.

[7] IEEE Standard for Local and Metropolitan Area Networks Part 16. Air Interface for Fixed and Mobile Broadband Wireless Access Systems Amendment for Physical and Medium Access Control Layers for Combined Fixed and Mobile Operation in Licensed Bands, 802.16E2005\&802.16/COR1, 2/2006.

[8] B. Muquet et al., "Cyclic prefixing or zero padding for wireless multicarrier transmissions?," IEEE Trans. Commun., vol. 50, no. 12, pp. 2136-2148, Dec. 2002.
[9] H. Steendam and M. Moeneclaey, "Different guard interval techniques for OFDM: Performance comparison," in Proc. MC-SS, Herrsching, Germany, May 2007, pp. 11-24.

[10] S. Tang et al., "Iterative channel estimation for block transmission with known symbol padding - A new look at TDS-OFDM," in Proc. IEEE Globecom, Washington, DC, USA, Nov. 2007, pp. 4269-4273.

[11] Website on unique word OFDM [Online]. Available: http://uwofdm. aau.at/

[12] A. Onic and M. Huemer, "Direct vs. two-step approach for unique word generation in UW-OFDM," in Proc. InOWo, Hamburg, Germany, Sep. 2010, pp. 145-149.

[13] C. Hofbauer, M. Huemer, and J. B. Huber, "Coded OFDM by unique word prefix," presented at the ICCS, Singapore, Nov. 2010.

[14] I. S. Reed and G. Solomon, "Polynomial codes over certain finite fields," J. SIAM, vol. 8, no. 2, pp. 300-304, 1960.

[15] W. Y. Zou and Y. Wu, "COFDM: An overview," IEEE Trans. Broadcast., vol. 41, no. 1, pp. 1-8, Mar. 1995.

[16] Z. Liu, Y. Xin, and G. B. Giannakis, "Linear constellation precoding for OFDM with maximum multipath diversity and coding gains," IEEE Trans. Commun., vol. 51, no. 3, pp. 416-427, Mar. 2003.

[17] Y.-P. Lin and S.-M. Phoong, "BER minimized OFDM systems with channel independent precoders," IEEE Trans. Signal Process., vol. 51, no. 9 , pp. 2369-2380, Sep. 2003

[18] D. Van Welden, "Channel Estimation and Synchronization for KSPOFDM," Ph.D. Thesis, Ghent Univ., Gent, Belgium, 2012.

[19] H. Steendam, "On the redundant carrier distribution for UW-OFDM," in Proc. WCNC, Paris, France, Apr. 1-4, 2012, pp. 32-36.

[20] H. Steendam, "Analysis of the redundant energy in UW-OFDM," IEEE Trans. Commun., vol. 60, no. 6, pp. 1692-1701, Jun. 2012.

[21] S. Song and A. C. Singer, "Pilot-aided OFDM channel estimation in the presence of the guard band," IEEE Trans. Commun., vol. 55, no. 8, pp. 1459-1465, Aug. 2007.

[22] C. D. Meyer, Matrix Analysis and Applied Linear Algebra. Philadelphia, PA, USA: SIAM, 2000

[23] A. H. Land and A. G. Doig, "An automatic method of solving discrete programming problems," Econometrica, vol. 28, no. 3, pp. 497-520, 1960, doi:10.2307/1910129.

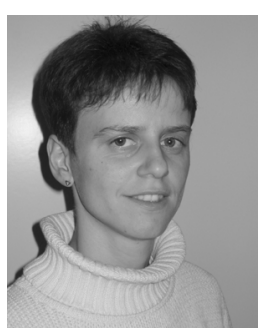

Heidi Steendam (M'01-SM'06) received the M.Sc. degree in electrical engineering and the Ph.D. degree in applied sciences from Ghent University, Gent, Belgium, in 1995 and 2000, respectively.

Since September 1995, she has been with the Digital Communications (DIGCOM) Research Group, Department of Telecommunications and Information Processing (TELIN), Faculty of Engineering, Ghen University, first in the framework of various research projects, and since October 2002, as a full-time Professor in the area of digital communications. Her main research interests are in statistical communication theory, carrier and symbol synchronization, bandwidth-efficient modulation and coding, spread-spectrum (multicarrier spread-spectrum), satellite and mobile communication, cognitive radio, and cooperative networks. She is the author of more than 100 scientific papers in international journals and conference proceedings.

Dr. Steendam has been an executive Committee Member of the IEEE Communications and Vehicular Technology Society Joint Chapter, Benelux Section, since 2002, and since 2012, is Vice Chair. She has been active in various international conferences as Technical Program Committee Chair/Member and Session Chair. In 2004 and 2011, she was the Conference Chair of the IEEE Symposium on Communications and Vehicular Technology in the Benelux. She is an Associate Editor of the IEEE TRANSACTIONS ON COMMUNICATIONS and EURASIP Journal on Wireless Communications and Networking. 